\title{
Accelerating Cosmologies in Lovelock Gravity with Dilaton
}

\author{
Ilya V. Kirnos* ${ }^{*}$ and Andrey N. Makarenko, ${ }^{2}$ \\ ${ }^{I}$ Tomsk State University, 634050, Tomsk, Lenin prosp., 36, Russia \\ ${ }^{2}$ Tomsk State Pedagogical University, 634041, Tomsk, Komsomol'sky prosp., 75, Russia
}

\begin{abstract}
For the description of the Universe expansion, compatible with observational data, a model of Lovelock gravity with dilaton is investigated. D-dimensional space with 3- and (D-4)-dimensional maximally symmetric subspaces is considered. Space without matter and space with perfect fluid are under test. In various forms of the theory (third order without dilaton and second order with dilaton and without it) there are obtained stationary, power-law, exponential and exponent-of-exponent form cosmological solutions. Last two forms include solutions which are clear to describe accelerating expansion of 3-dimensional subspace. Also there is a set of solutions describing cosmological expansion which does not tend to isotropization in the presence of matter.
\end{abstract}

Keywoerds: Cosmology, Dilation, Lovelock gravity.

\section{INTRODUCTION}

At present time there are numerous observational data known to be incompatible with the Standard Cosmological Model. On the one hand, accelerating expansion observations from supernovae type Ia [1] and gravitational lensing [2] allow us to calculate metric tensor. On the other hand, evaluating the amount of visible matter, energymomentum tensor can be obtained. However, it is impossible to satisfy Einstein equations by plugging in these values. Then there are two possibilities: there is a great amount of invisible matter or Einstein equations are not true. These possibilities point out two approaches to the problem: to develop theories of dark matter and dark energy or to modify theory of gravity.

In the present article we deal with the second approach. Modified gravity has its beginning in 1920-th. The most popular theories are Brans-Dicke theory [3, 4], Lovelock gravity [5] and $f(R)$-gravity (see, e. g. [6]). However, for a long time these theories were not useful for explanation of experimental data incompatible with general relativity.

Here we will investigate a scalar-tensor extension of Lovelock gravity --- Lovelock gravity with dilaton which might have its origin in low-energy limit of string theory. Lovelock gravity with dilaton contains scalar field $\varphi$ (dilaton), metric tensor $g_{\mu \nu}$, matter fields $\Phi^{I}$ and is described (in $D$-dimensional space-time) by Lagrangian where

$$
\begin{gathered}
m=\frac{1}{2} D, \quad \text { if } D \text { is even, } \\
m=\frac{1}{2}(D-1), \quad \text { if } D \text { is odd },
\end{gathered}
$$

$\alpha_{p}(\varphi), V(\varphi)$ are arbitrary functions of dilaton, $\delta_{v_{1} \cdots v_{k}}^{\mu_{1} \cdots \mu_{k}}$ is the generalized Kronecker delta which is equal to 1 if $v_{1} \cdots v_{k}$ is even transposition of $\mu_{1} \cdots \mu_{k}$, to -1 if odd one, and to zero otherwise; $\mathcal{L}_{M}\left(\Phi^{I}, g_{\mu v}\right)$ is the matter Lagrangian. We shall call terms

$$
\mathcal{L}_{p}=2 \delta_{\sigma_{1} \cdots \sigma_{2 p}}^{\lambda_{1} \cdots \lambda_{2 p}} R_{\lambda_{1} \lambda_{2}}^{\sigma_{1}^{\sigma_{2}}} R_{\lambda_{3} \lambda_{4}}^{\sigma_{3} \sigma_{4}} \cdots R_{\lambda_{2 p-1} \lambda_{2 p}}^{{ }^{\sigma_{2 p-1} \sigma_{2 p}}}
$$

as Lovelock Lagrangians of $p$-th order.

Usually (see, e. g. [7-9]) only 2-nd order (i. e. $\alpha_{p}=0$ $\forall p>2$ ) of Lovelock gravity without dilaton (so-called Einstein-Gauss-Bonnet gravity) is investigated. Solutions for more complicated variants of the theory are not large in number. Investigations of third order Lovelock gravity (without dilaton) can be found in [10-12], studies of the second order with dilaton can be found in [13-15]. Moreover, C. C. Briggs [16, 17] obtains explicit formulae for

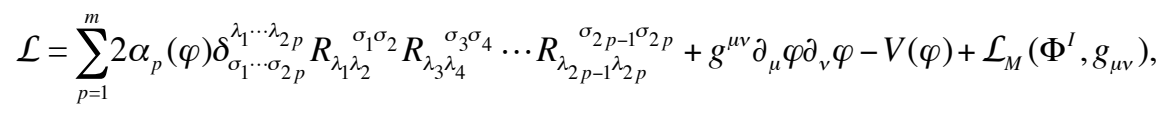

*Address correspondence to this author at the Tomsk State University, 634050, Russia, Tomsk, Lenin prosp., 36, Tel: +7 915 2374648; Fax: +7 (3822) 52-17-93; E-mail: ikirnos@mail.ru the 4-th and 5-th Lovelock tensors. Also we should draw attention to researches in $f\left(R, \mathcal{L}_{2}\right)$-gravity $[14,18]$ and to works in the 3-rd and 4-th orders of theory which is similar to Lovelock gravity and obtained from string theory lowenergy limit as well as the latter $[19,20]$. 


$$
\begin{aligned}
& G_{\mu v}^{(3)}=\frac{1}{2}\left(-g_{\mu v} R^{3}+12 g_{\mu v} R R_{\alpha \beta} R^{\alpha \beta}-3 g_{\mu v} R R_{\alpha \beta \sigma \kappa} R^{\alpha \beta \sigma \kappa}-16 g_{\mu v} R_{\alpha}{ }^{\beta} R_{\beta}{ }^{\sigma} R_{\sigma}{ }^{\alpha}+\right. \\
& +24 g_{\mu \nu} R_{\alpha \beta} R_{\sigma \kappa} R^{\alpha \sigma \beta \kappa}+24 g_{\mu v} R_{\alpha}{ }^{\beta} R^{\alpha \sigma \kappa \rho} R_{\beta \sigma \kappa \rho}+2 g_{\mu \nu} R_{\alpha \beta}{ }^{\sigma \kappa} R_{\sigma \kappa}{ }^{\rho \lambda} R_{\rho \lambda}{ }^{\alpha \beta}- \\
& -8 g_{\mu \nu} R_{\alpha \beta}{ }^{\sigma \kappa} R_{\sigma \rho}{ }^{\alpha \lambda} R_{\kappa \lambda}{ }^{\beta \rho}+6 R_{\mu v} R^{2}-24 R R_{\mu}{ }^{\sigma} R_{\sigma v}-24 R_{\mu \nu} R_{\alpha \beta} R^{\alpha \beta}+ \\
& +48 R_{\mu}{ }^{\alpha} R_{\alpha}{ }^{\beta} R_{\beta v}+48 R_{\mu}{ }^{\alpha} R^{\beta \sigma} R_{\alpha \beta \sigma v}+6 R_{\mu v} R_{\alpha \beta \sigma \kappa} R^{\alpha \beta \sigma \kappa}-24 R_{\mu \alpha} R_{v \beta \sigma \kappa} R^{\alpha \beta \sigma \kappa}+ \\
& +24 R R_{u \sigma v \kappa} R^{\sigma \kappa}+12 R R_{\mu \alpha \beta \sigma} R_{v}{ }^{\alpha \beta \sigma}-48 R_{\mu \alpha \nu \beta} R_{\sigma}{ }^{\alpha} R^{\sigma \beta}-48 R_{\mu \alpha \beta \sigma} R_{v}{ }^{\beta} R^{\alpha \sigma}+ \\
& +48 R_{\mu \alpha \nu \beta} R_{\sigma \kappa} R^{\alpha \sigma \beta \kappa}-24 R_{\mu \alpha \beta \sigma} R^{\kappa \alpha \beta \sigma} R_{\kappa v}-24 R_{\mu \alpha \beta \sigma} R^{\alpha \kappa} R_{v \kappa}^{\beta \sigma}+ \\
& +48 R_{\mu}{ }^{\alpha \beta \sigma} R_{\beta}{ }^{\kappa} R_{\text {бкv } \alpha}+24 R_{\mu \alpha \nu \beta} R^{\alpha}{ }_{\sigma \kappa \rho} R^{\beta \sigma \kappa \rho}+12 R_{\mu}{ }^{\alpha \beta \sigma} R_{\beta \sigma}{ }^{\kappa \rho} R_{\kappa \rho \alpha \nu}+ \\
& +48 R_{\mu \alpha}^{\beta \sigma} R_{\beta \rho \nu \lambda} R_{\sigma}^{\lambda \alpha \rho} \text { ). }
\end{aligned}
$$

In the present paper a set of solutions in second order with dilaton and without it, also in third order without dilaton are obtained. In the most of solutions extra spatial dimensions are assumed to exist. Unobservability of them is explained by Kaluza-Klein approach (see, e. g. [21, p. 186] and references therein) which is briefly the following: extra dimensions are compactified on so small scale that it is impossible to observe them (by present day device).

In the first section seven-dimensional third order Lovelock gravity without dilaton is considered. Second section is devoted to the second order with dilaton in spaces with various number of dimensions.

\section{THIRD ORDER LOVELOCK GRAVITY WITHOUT DILATON}

Because it seems impossible to consider general case of Lovelock gravity in a space with great number of dimensions, let us now discuss third order Lovelock gravity without dilaton and without cosmological constant: fields are $g_{\mu v}$ and $\Phi^{I}$, Lagrangian is

$$
\mathcal{L}_{\text {Lovelock } 3}=R+\alpha_{2} \mathcal{L}_{2}+\alpha_{3} \mathcal{L}_{3}+\mathcal{L}_{M}\left(\Phi^{I}, g_{\mu v}\right) .
$$

Here $\alpha_{2}, \alpha_{3}$ are constants. General expressions for 2nd and 3-rd Lovelock Lagrangians are:

$$
\begin{aligned}
& \mathcal{L}_{2}=R_{\mu v \alpha \beta} R^{\mu \nu \alpha \beta}-4 R_{\mu \nu} R^{\mu v}+R^{2}
\end{aligned}
$$

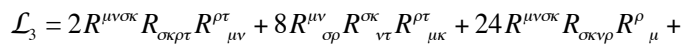

$$
\begin{aligned}
& +3 R R^{\mu v \sigma \kappa} R_{\mu v \sigma \kappa}+24 R^{\mu v \sigma K} R_{o u} R_{k v}+16 R^{\mu v} R_{v \sigma} R_{\mu}^{\sigma}{ }_{\mu}-12 R R^{\mu v} R_{\mu v}+R
\end{aligned}
$$

is Gauss-Bonnet Lagrangian,

is third Lovelock Lagrangian.

By variation of action $S=\int \mathcal{L}_{\text {Lovelock } 3} d^{D} x$ one may obtain

$$
G_{\mu \nu}^{(1)}+\alpha_{2} G_{\mu \nu}^{(2)}+\alpha_{3} G_{\mu \nu}^{(3)}=\frac{8 \pi G}{c^{4}} T_{\mu \nu}
$$

where

$$
G_{\mu \nu}^{(1)}=R_{\mu v}-\frac{1}{2} R g_{\mu v},
$$

$$
\begin{aligned}
& G_{\mu \nu}^{(2)}=2\left(-R_{\mu \sigma \kappa \tau} R^{\kappa \tau \sigma}{ }_{v}-2 R_{\mu \rho v \sigma} R^{\rho \sigma}-2 R_{\mu \sigma} R_{v}^{\sigma}+R R_{\mu v}\right)- \\
& \frac{1}{2}\left(R_{\rho \sigma \alpha \beta} R^{\rho \sigma \alpha \beta}-4 R_{\alpha \beta} R^{\alpha \beta}+R^{2}\right) g_{\mu v},
\end{aligned}
$$

and $G_{\mu \nu}^{(3)}$ is written down according to [16]:

\subsection{Cosmological Equations}

Now consider seven-dimensional ${ }^{1}$ flat space and assume metric tensor to get the form

$$
g_{\mu v}=\operatorname{diag}\left\{-1, a^{2}(t), a^{2}(t), a^{2}(t), b^{2}(t), b^{2}(t), b^{2}(t)\right\} .
$$

Furthermore, let $T_{\mu v}=0$.

From such a metric one can obtain nonzero Christoffel symbols:

$$
\Gamma_{i i}^{0}=a \dot{a}, \quad \Gamma_{a a}^{0}=b \dot{b} \quad \Gamma_{i 0}^{i}=\Gamma_{0 i}^{i}=\frac{\dot{a}}{a}, \quad \Gamma_{c 0}^{c}=\Gamma_{0 c}^{c}=\frac{\dot{b}}{b}
$$

(Latin indexes from the middle of alphabet $\mathrm{i}, \mathrm{j}, \mathrm{k}, \ldots$ run over visible subspace, and Latin indexes from the beginning of alphabet $a, b, c, \ldots$ run over extra subspace; index 0 notice the time coordinate; Greek indexes run over all the space). Nonzero components of Riemann tensor are

$$
\begin{array}{ll}
R_{i 0 i}^{0}=a(t) \ddot{a}(t) ; & R_{c 0 c}^{0}=b(t) \ddot{b}(t) ; \\
R_{0 i 0}^{i}=-\frac{\ddot{a}(t)}{a(t)} ; & R_{0 c 0}^{c}=-\frac{\ddot{b}(t)}{b(t)}, \\
R_{j i j}^{i}=\dot{a}^{2}(t), \quad i \neq j ; & R^{c}{ }_{d c d}=\dot{b}^{2}(t), \quad c \neq d ; \\
R_{c i c}^{i}=\frac{\dot{a}(t)}{a(t)} b(t) \dot{b}(t) ; & R_{i c i}^{c}=\frac{\dot{b}(t)}{b(t)} a(t) \dot{a}(t) .
\end{array}
$$

Now the field equations (5) are

${ }^{1}$ We elected 7-dimensional space for two reasons. First, just in such a space nonzero third order of Lovelock gravity arises. Second, in such a space we obtain general exact solution of the present form for the second order theory (see below). 


$$
\left\{\begin{array}{l}
H^{2}+3 H h+h^{2}+12 \alpha_{2} H^{3} h+36 \alpha_{2} H^{2} h^{2}+12 \alpha_{2} H h^{3}-240 \alpha_{3} H^{3} h^{3}=0, \\
\dot{H}\left(2+24 \alpha_{2} H h+24 \alpha_{2} h^{2}-288 \alpha_{3} H h^{3}\right)+\dot{h}\left(3+48 \alpha_{2} H h+12 \alpha_{2} H^{2}+\right. \\
\left.\quad+12 \alpha_{2} h^{2}-432 \alpha_{3} H^{2} h^{2}\right)+3 H^{2}+6 H h+6 h^{2}+72 \alpha_{2} H^{2} h^{2}+ \\
\quad+72 \alpha_{2} H h^{3}+24 \alpha_{2} H^{3} h+12 \alpha_{2} h^{4}-432 \alpha_{3} H^{2} h^{4}-288 \alpha_{3} H^{3} h^{3}=0, \\
\quad \\
\left.\quad+38 \alpha_{2} H h+12 \alpha_{2} h^{2}+12 \alpha_{2} H^{2}-432 \alpha_{3} H^{2} h^{2}\right)+\dot{h}\left(2+24 \alpha_{2} H h+\right. \\
\left.\quad+24 \alpha_{2} H^{2}-288 \alpha_{3} H^{3} h\right)+3 h^{2}+6 H h+6 H^{2}+72 \alpha_{2} H^{2} h^{2}+ \\
\quad+72 \alpha_{2} H^{3} h+24 \alpha_{2} H h^{3}+12 \alpha_{2} H^{4}-432 \alpha_{3} H^{4} h^{2}-288 \alpha_{3} H^{3} h^{3}=0 .
\end{array}\right.
$$

Here

$$
H(t)=\dot{a}(t) / a(t), \quad h(t)=\dot{b}(t) / b(t)
$$

are Hubble parameters for visible and extra dimensions respectively.

Note that third equation is consequence of two other equations. A reader is asked to verify this correlation by himself.

It seems reasonable to begin consideration of system (11) with allowing $\alpha_{2}=\alpha_{3}=0$ and solving therefore 7dimensional Einstein equations. But all solutions of them are only particular cases of generalized Kasner solution (see [22] and [23, §117] for 4-dimensional case). Particularly, in our case (9) there is a solution

$$
\begin{aligned}
& a(t)=a^{(0)} \frac{1}{\left(t_{0}-t\right)^{\frac{3+\sqrt{5}}{6(\sqrt{5}+2)}}} \approx a^{(0)} \frac{1}{\left(t_{0}-t\right)^{0.206},} \\
& b(t)=b^{(0)}\left(t_{0}-t\right)^{\frac{3-\sqrt{5}}{6(\sqrt{5}-2)}} \approx b^{(0)}\left(t_{0}-t\right)^{0.539},
\end{aligned}
$$

where $a(t)$ describes accelerated expansion of visible subspace.

\subsection{General Solution in the Second Order}

Assume $\alpha_{3}=0$ in (11). Then we have

From the first equation there are 3 possibilities:

$$
H=-\frac{1}{12 \alpha_{2} h}, \quad H=-\frac{3-\sqrt{5}}{2} h, \quad H=-\frac{3+\sqrt{5}}{2} h .
$$

Second and third possibilities are satisfied in all cases ( $H>0$ if $h<0$ ), first one --- under $\alpha_{2}>0$. Consider them one by one.

1. $H=-\frac{1}{12 \alpha_{2} h}$. Plugging into the second equation we have

$$
\dot{h}=-\frac{1728 \alpha_{2}^{3} h^{6}+1}{12 \alpha_{2}\left(12 \alpha_{2} h^{2}+144 \alpha_{2}^{2} h^{4}+1\right)} .
$$

Then

$$
h=\frac{1}{6} \sqrt{\frac{3}{\alpha_{2}}} x
$$

where $x$ obeys equation

$$
x^{5}+3 \gamma x^{4}-5 x^{3}-5 \gamma x^{2}+3 x+\gamma=0,
$$

with

$$
\gamma=\tan \frac{\sqrt{3}\left(t+t_{0}\right)}{2 \sqrt{\alpha_{2}}}
$$

2. $H=-\frac{3-\sqrt{5}}{2} h$. Plugging into the second equation we have

$$
\dot{h}=\frac{3 h^{2}\left(-40 \alpha_{2} h^{2}+16 \alpha_{2} h^{2} \sqrt{5}-\sqrt{5}+5\right)}{2\left(-\sqrt{5}+18 \alpha_{2} h^{2} \sqrt{5}-30 \alpha_{2} h^{2}\right)} .
$$

Then $h$ obeys equation

$8 \operatorname{arctanh}\left(-\sqrt{\alpha_{2}} h+\sqrt{5 \alpha_{2}} h\right) \sqrt{\alpha_{2}} h+1-6\left(t+t_{0}\right) h+\sqrt{5}=0$.

3. $H=-\frac{3+\sqrt{5}}{2}$ Plugging into the second equation we have

$$
\begin{aligned}
& H^{2}+3 H h+h^{2}+12 \alpha_{2} H^{3} h+36 \alpha_{2} H^{2} h^{2}+12 \alpha_{2} H h^{3}=0 \\
& \dot{H}\left(2+24 \alpha_{2} H h+24 \alpha_{2} h^{2}\right)+\dot{h}\left(3+48 \alpha_{2} H h+12 \alpha_{2} H^{2}+\right. \\
& \left.\quad+12 \alpha_{2} h^{2}\right)+3 H^{2}+6 H h+6 h^{2}+72 \alpha_{2} H^{2} h^{2}+ \\
& \quad+72 \alpha_{2} H h^{3}+24 \alpha_{2} H^{3} h+12 \alpha_{2} h^{4}=0 \\
& \quad \\
& \quad \dot{H}\left(3+48 \alpha_{2} H h+12 \alpha_{2} h^{2}+12 \alpha_{2} H^{2}\right)+h\left(2+24 \alpha_{2} H h+\right. \\
& \left.\quad+24 \alpha_{2} H^{2}\right)+3 h^{2}+6 H h+6 H^{2}+72 \alpha_{2} H^{2} h^{2}+ \\
& \quad+72 \alpha_{2} H^{3} h+12 \alpha_{2} H^{4}=0 .
\end{aligned}
$$




$$
\dot{h}=\frac{3 h^{2}\left(40 \alpha_{2} h^{2}-5-\sqrt{5}+16 \alpha_{2} h^{2} \sqrt{5}\right)}{2\left(30 \alpha_{2} h^{2}+18 \alpha_{2} h^{2} \sqrt{5}-\sqrt{5}\right)} .
$$

Then $h$ obeys equation

$8 \operatorname{arctanh}\left(\sqrt{\alpha_{2}} h+\sqrt{5 \alpha_{2}} h\right) \sqrt{\alpha_{2}} h-1+6\left(t+t_{0}\right) h+\sqrt{5}=0$.

In all cases above there is only parametric dependence $H(t)$ and $h(t)$. Some explicit solutions in Einstein-GaussBonnet gravity for 7 or other dimensions will be obtained in the second section.

\subsection{Exponential Solution in the Third Order}

Now consider equations of the third Lovelock gravity with constant Hubble parameters:

$$
H=0, \quad h=0 .
$$

Then the system (11) takes a form

$$
H=\frac{1}{\sqrt{2 \alpha_{2}}}, \quad h=-\frac{1}{\sqrt{2 \alpha_{2}}} .
$$

Therefore (because of (12)),

$$
a(t)=C_{1} \exp \left(\frac{t}{\sqrt{2 \alpha_{2}}}\right), \quad b(t)=C_{2} \exp \left(-\frac{t}{\sqrt{2 \alpha_{2}}}\right)
$$

where $C_{1}, C_{2}$ are arbitrary positive constants.

It is clear that $\dot{a}(t)>0, \quad \ddot{a}(t)>0, \quad$ i. $\quad$ e. the abovementioned solution describes accelerated expansion of visible dimensions. At that extra dimensions shrink. Then it is possible that visible and extra dimensions were equivalent and the Universe look as 4-dimensional one only after expansion of one subspace and contraction of another one.

$$
\left\{\begin{aligned}
H^{2}+3 H h+h^{2}+12 \alpha_{2} H^{3} h+36 \alpha_{2} H^{2} h^{2}+ & 12 \alpha_{2} H h^{3}-240 \alpha_{3} H^{3} h^{3}=0 \\
3 H^{2}+6 H h+6 h^{2}+72 \alpha_{2} H^{2} h^{2}+72 \alpha_{2} H h^{3}+ & 24 \alpha_{2} H^{3} h+12 \alpha_{2} h^{4}- \\
& -432 \alpha_{3} H^{2} h^{4}-288 \alpha_{3} H^{3} h^{3}=0 \\
3 h^{2}+6 H h+6 H^{2}+72 \alpha_{2} H^{2} h^{2}+72 \alpha_{2} H^{3} h+ & 24 \alpha_{2} H h^{3}+12 \alpha_{2} H^{4}- \\
& -432 \alpha_{3} H^{4} h^{2}-288 \alpha_{3} H^{3} h^{3}=0
\end{aligned}\right.
$$

Subtracting the second equation from the third one we have

$$
\left(H^{2}-h^{2}\right)\left[1+16 \alpha_{2} H h+4 \alpha_{2}\left(H^{2}+h^{2}\right)-144 \alpha_{3} H^{2} h^{2}\right]=0 \text {. }
$$

Then $\mathrm{h}=-\mathrm{H}$ is a solution of this equation.

Plugging this equality into the second equation of (15) one can obtain 4 various solutions:

$$
H= \pm \sqrt{\frac{-\alpha_{2} \pm \sqrt{\alpha_{2}^{2}+12 \alpha_{3}}}{24 \alpha_{3}}}
$$

Because of the expansion of visible dimensions let us take only $H>0$. Then we have

$H=\sqrt{\frac{-\alpha_{2} \pm \sqrt{\alpha_{2}^{2}+12 \alpha_{3}}}{24 \alpha_{3}}}, \quad h=-\sqrt{\frac{-\alpha_{2} \pm \sqrt{\alpha_{2}^{2}+12 \alpha_{3}}}{24 \alpha_{3}}}$ obtain

Plugging $h=-H$ into the first equation of (15) we

$$
H= \pm \sqrt{\frac{-\alpha_{2} \pm \sqrt{\alpha_{2}^{2}+(20 / 3) \alpha_{3}}}{40 \alpha_{3}}} .
$$

Comparing that with (16) we have

$$
\alpha_{3}=-\frac{1}{12} \alpha_{2}^{2} \text {. }
$$

Then
Solution (17) seems to be useful for the description of inflation.

Finally, if $T_{\mu \nu}=0, \alpha_{2}>0$ and $\alpha_{3}=-\frac{1}{12} \alpha_{2}^{2}$ then system (5) has solution (9) where functions $a(t)$ and $b(t)$ are expressed by (19) with arbitrary positive constants $C_{1}$ and $C_{2}$.

\section{EINSTEIN-GAUSS-BONNET GRAVITY WITH DILATON}

Such a theory is the following: fields $g_{\mu \nu}, \varphi, \Phi^{I}$; Lagrangian

$$
\mathcal{L}_{E G B d}=R+g^{\mu v} \partial_{\mu} \varphi \partial_{v} \varphi-V(\varphi)+\varepsilon(\varphi) \mathcal{L}_{2}+\mathcal{L}_{M}\left(\Phi^{I}, g_{\mu v}\right)
$$

Here $\varepsilon(\varphi), V(\varphi)$ are functions of dilaton $\varphi, \mathcal{L}_{2}$ is a Gauss-Bonnet Lagrangian (3). Theory under consideration is different from generalized Brans-Dicke theory (see, e. g. [21]) even in 4-dimensional space, that is why we investigate both (3+1)-dimensional space and spaces with extra dimensions (where Einstein-Gauss-Bonnet gravity without dilaton is sensible).

Variating the action with Lagrangian (20) we get field equations: 


$$
\left\{\begin{array}{l}
R_{\mu \nu}-\frac{1}{2} R g_{\mu \nu}-\frac{1}{2} g_{\mu \nu} g^{\alpha \beta} \partial_{\alpha} \varphi \partial_{\beta} \varphi+\frac{1}{2} g_{\mu \nu} V(\varphi)+\varepsilon(\varphi) G_{\mu \nu}^{(2)}+\partial_{\mu} \varphi \partial_{\nu} \varphi- \\
\quad-4\left(R_{\mu \nu}-\frac{1}{2} R g_{\mu \nu}\right) \square \varepsilon(\varphi)-4\left(R_{\mu}{ }^{\alpha \beta}{ }_{\nu}+R^{\alpha \beta} g_{\mu \nu}\right) \nabla_{\alpha} \nabla_{\beta} \varepsilon(\varphi)+ \\
\quad+8 R^{\alpha}{ }_{\mu} \nabla_{\alpha} \nabla_{\nu} \varepsilon(\varphi)-2 R \nabla_{\mu} \nabla_{\nu} \varepsilon(\varphi)=\frac{8 \pi G}{c^{4}} T_{\mu \nu} \\
2 \square \varphi+V^{\prime}(\varphi)-\varepsilon^{\prime}(\varphi) \mathcal{L}_{2}=0 .
\end{array}\right.
$$

Here $G_{\mu v}^{(2)}$ is the second Lovelock tensor (7).

\subsection{Cosmological Equations}

Consider space of $D=p+q+1$ dimensions with two maximally symmetric subspaces: $p$-dimensional and $q$ dimensional. Square interval in such a space is

$$
d s^{2}=-e^{2 u_{0}(t)} d t^{2}+e^{2 u_{1}(t)} d s_{p}^{2}+e^{2 u_{2}(t)} d s_{q}^{2},
$$

where $d s_{p}^{2}$ and $d s_{q}^{2}$ are square intervals in $p$ - and $q$ dimensional subspaces respectively, $u_{0}(t), u_{1}(t), u_{2}(t)$ are arbitrary functions of time $t$.

If metric is (22) then Christoffel symbols are (as above, Latin indexes from the middle of alphabet $\mathrm{i}, \mathrm{j}, \mathrm{k}, \ldots$ run over visible $p$-subspace, and Latin indexes from the beginning of alphabet a,b,c,... run over extra $q$-subspace; index 0 notice the time coordinate; Greek indexes run over all the space)

$$
\begin{aligned}
& \Gamma_{00}^{0}=\dot{u}_{0}, \quad \Gamma_{i j}^{0}=\dot{u}_{1} e^{-2 u_{0}} g_{i j}, \quad \Gamma_{a b}^{0}=\dot{u}_{2} e^{-2 u_{0}} g_{a b}, \\
\Gamma_{j k}^{i}= & \tilde{\Gamma}_{j k}^{i}, \quad \Gamma_{0 i}^{i}=\Gamma_{i 0}^{i}=\dot{u}_{1}, \quad \Gamma_{b c}^{a}=\tilde{\Gamma}_{b c}^{a}, \quad \Gamma_{0 a}^{a}=\Gamma_{a 0}^{a}=\dot{u}_{2} .
\end{aligned}
$$

Riemann tensor, Ricci tensor and scalar curvature are

$$
\begin{aligned}
& R_{i 0 j}^{0}=e^{-2 u_{0}} X g_{i j}, \quad R_{a 0 b}^{0}=e^{-2 u_{0}} Y g_{a b}, \\
& R_{j k l}^{i}=e^{-2 u_{0}} A_{p}\left(\delta_{k}^{i} g_{j l}-\delta_{l}^{i} g_{j k}\right), \quad R_{a j b}^{i}=e^{-2 u_{0}} \dot{u}_{1} \dot{u}_{2} \delta_{j}^{i} g_{a b}, \\
& R_{b c d}^{a}=e^{-2 u_{0}} A_{q}\left(\delta_{c}^{a} g_{b d}-\delta_{d}^{a} g_{b c}\right), \quad R_{00}=-p X-q Y,
\end{aligned}
$$

$$
\begin{aligned}
& R_{i j}=e^{-2 u_{0}}\left(X+(p-1) A_{p}+q \dot{u}_{1} \dot{u}_{2}\right) g_{i j}, \\
& R_{a b}=e^{-2 u_{0}}\left(Y+(q-1) A_{q}+p \dot{u}_{1} \dot{u}_{2}\right) g_{a b}, \\
& R=e^{-2 u_{0}}\left[2 p X+2 q Y+p_{1} A_{p}+q_{1} A_{q}+2 p q \dot{u}_{1} \dot{u}_{2}\right] .
\end{aligned}
$$

Gauss - Bounnet Lagrangian (3)

Here we introduce the following notations:

$$
\begin{gathered}
A_{p} \equiv \dot{u}_{1}^{2}+\sigma_{p} e^{2\left(u_{0}-u_{1}\right)}, \quad A_{q} \equiv \dot{u}_{2}^{2}+\sigma_{q} e^{2\left(u_{0}-u_{2}\right)}, \\
X \equiv \ddot{u}_{1}-\dot{u}_{0} \dot{u}_{1}+\dot{u}_{1}^{2}, \quad Y \equiv \ddot{u}_{2}-\dot{u}_{0} \dot{u}_{2}+\dot{u}_{2}^{2}, \\
(p-m)_{n} \equiv(p-m)(p-m-1)(p-m-2) \ldots(p-n), \\
(q-m)_{n} \equiv(q-m)(q-m-1)(q-m-2) \ldots(q-n), \\
\sigma_{p} \equiv \frac{\tilde{R}_{p}}{p(p-1)} \quad \sigma_{q} \equiv \frac{\tilde{R}_{q}}{q(q-1)},
\end{gathered}
$$

where $\tilde{R}_{p}$ and $\tilde{R}_{q}$ are internal curvatures of $p$ - and $q$ dimensional subspaces respectively, $\tilde{\Gamma}_{j k}^{i}$ and $\tilde{\Gamma}_{b c}^{a}$ are internal Christoffel symbols.

Now field equations are

These equations are equivalent to those in [15] if we substitute $g^{\mu v} \partial_{\mu} \varphi \partial_{\nu} \varphi$ by $-\frac{1}{2} g^{\mu v} \partial_{\mu} \varphi \partial_{\nu} \varphi$ in Lagrangian and

$$
\begin{aligned}
& \mathcal{L}_{2}=e^{-4 u_{0}}\left\{p_{3} A_{p}^{2}+2 p_{1} q_{1} A_{p} A_{q}+q_{3} A_{q}^{2}+4 \dot{u}_{1} \dot{u}_{2}\left(p_{2} q A_{p}+p q_{2} A_{q}\right)+4 p_{1} q_{1} \dot{u}_{1}^{2} \dot{u}_{2}^{2}+\right. \\
&+4 p X {\left.\left[(p-1)_{2} A_{p}+q_{1} A_{q}+2(p-1) q \dot{u}_{1} \dot{u}_{2}\right]+4 q Y\left[p_{1} A_{p}+(q-1)_{2} A_{q}+2 p(q-1) \dot{u}_{1} \dot{u}_{2}\right]\right\} } \\
& \frac{1}{2} p_{1} A_{p}+\frac{1}{2} q_{1} A_{q}+p q \dot{u}_{1} \dot{u}_{2}+\frac{1}{2} \dot{\varphi}^{2}-\frac{1}{2} e^{-2 u_{0}} V(\varphi)+\frac{1}{2} e^{-2 u_{0}} \varepsilon(\varphi)\left\{p_{3} A_{p}^{2}+2 p_{1} q_{1} A_{p} A_{q}+\right. \\
&\left.\quad+q_{3} A_{q}^{2}+4 \dot{u}_{1} \dot{u}_{2}\left(p_{2} q A_{p}+p q_{2} A_{q}\right)+4 p_{1} q_{1} \dot{u}_{1}^{2} \dot{u}_{2}^{2}\right\}+2 e^{-2 u_{0}} \varepsilon^{\prime}(\varphi) \dot{\varphi}\left\{A _ { p } \left(p_{2} q \dot{u}_{1}+\right.\right. \\
&\left.\left.+q p_{1} \dot{u}_{2}\right)+A_{q}\left(p q_{1} \dot{u}_{1}+q_{2} \dot{u}_{2}\right)+2 \dot{u}_{1} \dot{u}_{2}\left(p_{1} q \dot{u}_{1}+p q_{1} \dot{u}_{2}\right)\right\}=\frac{8 \pi G}{c^{4}} T_{00},
\end{aligned}
$$




$$
\begin{aligned}
& e^{-2 u_{0}}\left\{(1-p) X-q Y-\frac{1}{2}(p-1)_{2} A_{p}-\frac{1}{2} q_{1} A_{q}-(p-1) q \dot{u}_{1} \dot{u}_{2}\right\} g_{i j}+\frac{1}{2} e^{-2 u_{0}} \dot{\varphi}^{2} g_{i j}+ \\
& +\frac{1}{2} V(\varphi) g_{i j}-\frac{1}{2} \varepsilon(\varphi) e^{-4 u_{0}} g_{i j}\left\{(p-1)_{4} A_{p}^{2}+4(p-1)_{2} q_{1} \dot{u}_{1}^{2} \dot{u}_{2}^{2}+4(p-1)_{3} A_{p} X+\right. \\
& +4(p-1)_{3} q \dot{u}_{1} \dot{u}_{2} A_{p}+8(p-1) q_{1} \dot{u}_{1} \dot{u}_{2} Y+4(p-1) q_{2} \dot{u}_{1} \dot{u}_{2} A_{q}+4(p-1) q_{1} A_{q} X+ \\
& \left.+8(p-1)_{2} q \dot{u}_{1} \dot{u}_{2} X+4(p-1)_{2} q A_{p} Y+2(p-1)_{2} q_{1} A_{p} A_{q}+q_{3} A_{q}^{2}+4 q_{2} A_{q} Y\right\}+ \\
& +2 e^{-4 u_{0}} g_{i j}\left\{-\left(\varepsilon^{\prime \prime} \dot{\varphi}^{2}+\varepsilon^{\prime} \ddot{\varphi}-\varepsilon^{\prime} \dot{u}_{0} \dot{\varphi}\right)\left[(p-1)_{2} A_{p}+q_{1} A_{q}+2(p-1) q \dot{u}_{1} \dot{u}_{2}\right]+\right. \\
& +\varepsilon^{\prime} \dot{u}_{1} \dot{\varphi}\left[-2(p-1)_{2} X-2(p-1) q Y-(p-1)_{3} A_{p}-(p-1) q_{1} A_{q}-\right. \\
& \left.-2(p-1)_{2} q \dot{u}_{1} \dot{u}_{2}\right]+\varepsilon^{\prime} \dot{u}_{2} \dot{\varphi}\left[-2(p-1) q X-2 q_{1} Y-(p-1)_{2} q A_{p}-\right. \\
& \left.\left.-q_{2} A_{q}-2(p-1) q_{1} \dot{u}_{1} \dot{u}_{2}\right]\right\}=\frac{8 \pi G}{c^{4}} T_{i j} \text {, } \\
& e^{-2 u_{0}}\left\{(1-q) Y-p X-\frac{1}{2}(q-1)_{2} A_{q}-\frac{1}{2} p_{1} A_{p}-(q-1) p \dot{u}_{1} \dot{u}_{2}\right\} g_{a b}+\frac{1}{2} e^{-2 u_{0}} \dot{\varphi}^{2} g_{a b}+ \\
& +\frac{1}{2} V(\varphi) g_{a b}-\frac{1}{2} \varepsilon(\varphi) e^{-4 u_{0}} g_{a b}\left\{(q-1)_{4} A_{q}^{2}+4(q-1)_{2} p_{1} \dot{u}_{1}^{2} \dot{u}_{2}^{2}+4(q-1)_{3} A_{q} Y+\right. \\
& +4(q-1)_{3} p \dot{u}_{1} \dot{u}_{2} A_{q}+8(q-1) p_{1} \dot{u}_{1} \dot{u}_{2} X+4(q-1) p_{2} \dot{u}_{1} \dot{u}_{2} A_{p}+4(q-1) p_{1} A_{p} Y+ \\
& \left.+8(q-1)_{2} p \dot{u}_{1} \dot{u}_{2} Y+4(q-1)_{2} p A_{q} X+2(q-1)_{2} p_{1} A_{p} A_{q}+p_{3} A_{p}^{2}+4 p_{2} A_{p} X\right\}+ \\
& +2 e^{-4 u_{0}} g_{a b}\left\{-\left(\varepsilon^{\prime \prime} \dot{\varphi}^{2}+\varepsilon^{\prime} \ddot{\varphi}-\varepsilon^{\prime} \dot{u}_{0} \dot{\varphi}\right)\left[(q-1)_{2} A_{q}+p_{1} A_{p}+2(q-1) p \dot{u}_{1} \dot{u}_{2}\right]+\right. \\
& +\varepsilon^{\prime} \dot{u}_{2} \dot{\varphi}\left[-2(q-1)_{2} Y-2(q-1) p X-(q-1)_{3} A_{q}-(q-1) p_{1} A_{p}-\right. \\
& \left.-2(q-1)_{2} p \dot{u}_{1} \dot{u}_{2}\right]+\varepsilon^{\prime} \dot{u}_{1} \dot{\varphi}\left[-2(q-1) p Y-2 p_{1} X-(q-1)_{2} p A_{q}-\right. \\
& \left.\left.-p_{2} A_{p}-2(q-1) p_{1} \dot{u}_{1} \dot{u}_{2}\right]\right\}=\frac{8 \pi G}{c^{4}} T_{a b} \text {, } \\
& 2\left[\ddot{\varphi}+\left(-\dot{u}_{0}+p \dot{u}_{1}+q \dot{u}_{2}\right) \dot{\varphi}\right]-V^{\prime}(\varphi)+\varepsilon^{\prime}(\varphi) e^{-2 u_{0}}\left\{p_{3} A_{p}^{2}+2 p_{1} q_{1} A_{p} A_{q}+q_{3} A_{q}^{2}+\right. \\
& +4 \dot{u}_{1} \dot{u}_{2}\left(p_{2} q A_{p}+p q_{2} A_{q}\right)+4 p_{1} q_{1} \dot{u}_{1}^{2} \dot{u}_{2}^{2}+4 p X\left[(p-1)_{2} A_{p}+q_{1} A_{q}+\right. \\
& \left.\left.+2(p-1) q \dot{u}_{1} \dot{u}_{2}\right]+4 q Y\left[p_{1} A_{p}+(q-1)_{2} A_{q}+2 p(q-1) \dot{u}_{1} \dot{u}_{2}\right]\right\}=0 \text {. }
\end{aligned}
$$

put $\varepsilon(\varphi)=\alpha_{2} e^{-\gamma \varphi}, V(\varphi)=0, T_{\mu \nu}=0$.

Henceforth, we may put $u_{0}=0$ (for simplification), $p=3$ (to identify $p$-subspace with visible space).

\subsection{Stationary Solutions}

Let us now turn to find solutions of (27)-(30) under $p=3, u_{0}=0$. The simplest solutions are stationary ones. Hence put

$$
u_{1}=\text { const }, \quad u_{2}=\text { const }, \quad \varphi=\text { const } .
$$

Then (24) are

$$
A_{p}=\sigma_{p} e^{-2 u_{1}}, \quad A_{q}=\sigma_{q} e^{-2 u_{2}}, \quad X=Y=0 .
$$

Consider space with homogeneous dust, i. e. $T_{00} \neq 0$ (other $T_{\mu v}=0$ ).

After that, system (27)-(30) get the form of algebraic equations

$$
3 A_{p}+\frac{1}{2} q_{1} A_{q}-\frac{1}{2} V(\varphi)+\frac{1}{2} \varepsilon(\varphi)\left\{12 q_{1} A_{p} A_{q}+q_{3} A_{q}^{2}\right\}=\frac{8 \pi G}{c^{4}} T_{00} ;
$$

$$
\begin{gathered}
-A_{p}-\frac{1}{2} q_{1} A_{q}+\frac{1}{2} V(\varphi)-\frac{1}{2} \varepsilon(\varphi)\left\{4 q_{1} A_{q} A_{p}+q_{3} A_{q}^{2}\right\}=0 \\
-\frac{1}{2}(q-1)_{2} A_{q}-3 A_{p}+\frac{1}{2} V(\varphi)-\frac{1}{2} \varepsilon(\varphi) \\
\left\{(q-1)_{4} A_{q}^{2}+12(q-1)_{2} A_{p} A_{q}\right\}=0 \\
V^{\prime}(\varphi)-\varepsilon^{\prime}(\varphi)\left\{12 q_{1} A_{p} A_{q}+q_{3} A_{q}^{2}\right\}=0 .
\end{gathered}
$$

From (34) and (31) we have

$$
3 A_{p}+\frac{1}{2} q_{1} A_{q}=\frac{1}{2} V(\varphi)-\frac{1}{2} \varepsilon(\varphi) \frac{V^{\prime}(\varphi)}{\varepsilon^{\prime}(\varphi)}+\frac{8 \pi G}{c^{4}} T_{00} .
$$

Try to find linear combination of (31)-(33) in order to cancel terms with $\varepsilon(\varphi)$. Let $\alpha, \beta, \gamma$ are coefficients for (32), (33) and (31) in such a combination. Then we need

$$
\left\{\begin{array}{l}
\alpha q_{3}+\beta(q-1)_{4}=\gamma q_{3}, \\
4 \alpha q_{1}+12 \beta(q-1)_{2}=12 \gamma q_{1} .
\end{array}\right.
$$

Therefore

$$
\gamma=\left(1-\frac{1}{q}\right) \beta, \quad \alpha=\frac{3}{q} \beta
$$


Now put $\beta=q$. Then $\alpha=3, \gamma=q-1$.

Now multiplying (32) by $3 /(\mathrm{q}+1)$ and putting them together we have (taking (34) into account).

$3 A_{p}+\frac{1}{2} q_{1} A_{q}=\frac{q+3}{2(q+1)} V(\varphi)-\frac{q-1}{2(q+1)} \varepsilon(\varphi) \frac{V^{\prime}(\varphi)}{\varepsilon^{\prime}(\varphi)}$.

From this and (35) one can get

$$
\frac{1}{q+1} V(\varphi)+\frac{1}{q+1} \varepsilon(\varphi) \frac{V^{\prime}(\varphi)}{\varepsilon^{\prime}(\varphi)}-\frac{8 \pi G}{c^{4}} T_{00}=0 .
$$

Put now

$$
V(\varphi)=a e^{-\alpha \varphi}, \quad \varepsilon(\varphi)=b e^{-\beta \varphi} .
$$

Then (37) get the form

$$
\frac{1}{q+1}\left(1+\frac{\alpha}{\beta}\right) a e^{-\alpha \varphi}-\frac{8 \pi G}{c^{4}} T_{00}=0 .
$$

It is easy to see:

$$
\varphi=-\frac{1}{\alpha} \ln \left\{\frac{\beta(q+1)}{a(\alpha+\beta)} \cdot \frac{8 \pi G}{c^{4}} T_{00}\right\} .
$$

Plugging this $\varphi$ into (35) we have:

$$
3 A_{p}+\frac{1}{2} q_{1} A_{q}=\frac{4 \pi G}{c^{4}} T_{00} \frac{(1-q) \alpha+(q+3) \beta}{\alpha+\beta} .
$$

Plugging $A_{p}$ derived from that into (34) we can get

$$
\begin{aligned}
& A_{q}^{2} q_{1}\{(q-2)(q-3)-2 q(q-1)\}+ \\
& 2 q_{1} \chi \frac{(1-q) \alpha+(q+3) \beta}{\alpha+\beta} A_{q}=\frac{\alpha a}{\beta b}\left\{\frac{\beta(q+1)}{a(\alpha+\beta)} \chi\right\}^{\frac{\alpha-\beta}{\alpha}},
\end{aligned}
$$

where

$$
\chi \equiv \frac{8 \pi G}{c^{4}} T_{00} .
$$

That is quadratic equation on $A_{q}$, which solutions are

$$
A_{q}=\frac{-2 q_{1} \chi \frac{(1-q) \alpha+(q+3) \beta}{\alpha+\beta} \pm \sqrt{D}}{2\left(q_{3}-2 q_{1}^{2}\right)},
$$

where
Then, taking (39) into account, we obtain

$$
A_{p}=\frac{1}{6} \chi \frac{(1-q) \alpha+(q+3) \beta}{\alpha+\beta}-\frac{q_{1}}{6} A_{q} .
$$

Finally,

$$
u_{1}=-\frac{1}{2} \ln \frac{A_{p}}{\sigma_{p}}, \quad u_{2}=-\frac{1}{2} \ln \frac{A_{q}}{\sigma_{q}}
$$

and $\varphi$ is (38).

It should be noted that the following constraints was applied:

$$
\begin{aligned}
& T_{00} \neq 0, \quad a \neq 0, \quad b \neq 0, \quad \alpha \neq 0, \quad \beta \neq 0, \\
& \alpha \neq-\beta, \quad q \neq 0, \quad q \neq 2, \quad q \neq 4, \quad \sigma_{p} \neq 0, \quad \sigma_{q} \neq 0 .
\end{aligned}
$$

It is easy to derive solution for (3+1)-dimensional space with perfect fluid of arbitrary equation of state parameter. At that we should not specify $V(\varphi)$ and $\varepsilon(\varphi)$ because of $\varepsilon(\varphi)$ do not participate in equations and $V(\varphi)$ is specified from those. Dilaton also do not contribute in equations, therefore we should solve just Einstein equations with cosmological constant. Solution is

$$
V=(1+3 w) \frac{8 \pi G}{c^{4}} T_{00}, \quad u_{1}=-\frac{1}{2} \ln \frac{1+w}{2 \sigma_{p}} \frac{8 \pi G}{c^{4}} T_{00}
$$

Here $\mathrm{w}$ is equation of state parameter $(p=\mathcal{w} \varepsilon, p$ is pressure, $\varepsilon \equiv T_{00}$ is energy density). A particular case (when $\varepsilon=0$ ) was derived by A. Einstein in 1917 [24].

\subsection{Exponential Solutions}

For the dynamical solutions we need to do further simplification of (27)-(30). Therefore, in addition to $p=3$ and $u_{0}=$ 0 , put $\sigma_{p}=\sigma_{q}=0$ i. e. subspaces are flat. In addition to simplicity, such a condition is caused by Cosmic Microwave Background observations $[25,26]$ indicate the flatness of visible subspace. For extra subspace $\sigma_{q}=0$ is only a simplification.

After that, equations (27)-(30) are

$$
\begin{aligned}
D= & \left(2 q_{1} \varkappa \frac{(1-q) \alpha+(q+3) \beta}{\alpha+\beta}\right)^{2}+4 q_{1}\{(q-2)(q-3)-2 q(q-1)\} \frac{\alpha a}{\beta b}\left\{\frac{\beta(q+1)}{a(\alpha+\beta)} \varkappa\right\}^{\frac{\alpha-\beta}{\alpha}} . \\
3 \dot{u}_{1}^{2} & +\frac{1}{2} q_{1} \dot{u}_{2}^{2}+3 q \dot{u}_{1} \dot{u}_{2}+\frac{1}{2} \dot{\varphi}^{2}-\frac{1}{2} V(\varphi)+\frac{1}{2} \varepsilon(\varphi)\left\{36 q_{1} \dot{u}_{1}^{2} \dot{u}_{2}^{2}+q_{3} \dot{u}_{2}^{4}+12 \dot{u}_{1} \dot{u}_{2}\left(2 q \dot{u}_{1}^{2}+q_{2} \dot{u}_{2}^{2}\right)\right\}+ \\
& +2 \varepsilon^{\prime} \dot{\varphi}\left\{6 q \dot{u}_{1}^{3}+18 q \dot{u}_{1}^{2} \dot{u}_{2}+9 q_{1} \dot{u}_{1} \dot{u}_{2}^{2}+q_{2} \dot{u}_{2}^{3}\right\}=\frac{8 \pi G}{c^{4}} T_{00} .
\end{aligned}
$$




$$
\begin{aligned}
\{ & \left.-2 \ddot{u}_{1}-q \ddot{u}_{2}-3 \dot{u}_{1}^{2}-\frac{1}{2} q(q+1) \dot{u}_{2}^{2}-2 q \dot{u}_{1} \dot{u}_{2}\right\} g_{i j}+\frac{1}{2} \dot{\varphi}^{2} g_{i j}+\frac{1}{2} V(\varphi) g_{i j}- \\
& -\frac{1}{2} \varepsilon(\varphi) g_{i j}\left\{8 \ddot{u}_{1} \dot{u}_{2}\left(q_{1} \dot{u}_{2}+2 q \dot{u}_{1}\right)+4 \ddot{u}_{2}\left(4 q_{1} \dot{u}_{1} \dot{u}_{2}+2 q \dot{u}_{1}^{2}+q_{2} \dot{u}_{2}^{2}\right)+(q+1) q_{2} \dot{u}_{2}^{4}+\right. \\
& \left.+4 q(5 q-3) \dot{u}_{1}^{2} \dot{u}_{2}^{2}+8 q q_{1} \dot{u}_{1} \dot{u}_{2}^{3}+16 q \dot{u}_{1}^{3} \dot{u}_{2}\right\}+2 g_{i j}\left\{-\left(\varepsilon^{\prime \prime} \dot{\varphi}^{2}+\varepsilon^{\prime} \ddot{\varphi}\right)\left[2 \dot{u}_{1}^{2}+q_{1} \dot{u}_{2}^{2}+\right.\right. \\
& \left.+4 q \dot{u}_{1} \dot{u}_{2}\right]+\varepsilon^{\prime} \dot{u}_{1} \dot{\varphi}\left[-4 \ddot{u}_{1}-4 q \ddot{u}_{2}-4 \dot{u}_{1}^{2}-2(q+1) q \dot{u}_{2}^{2}-4 q \dot{u}_{1} \dot{u}_{2}\right]+ \\
& \left.+\varepsilon^{\prime} \dot{u}_{2} \dot{\varphi}\left[-4 q \ddot{u}_{1}-2 q_{1} \ddot{u}_{2}-6 q \dot{u}_{1}^{2}-q q_{1} \dot{u}_{2}^{2}-4 q_{1} \dot{u}_{1} \dot{u}_{2}\right]\right\}=\frac{8 \pi G}{c^{4}} T_{i j} . \\
\{- & \left.3 \ddot{u}_{1}-(q-1) \ddot{u}_{2}-6 \dot{u}_{1}^{2}-\frac{1}{2} q_{1} \dot{u}_{2}^{2}-3(q-1) \dot{u}_{1} \dot{u}_{2}\right\} g_{a b}+\frac{1}{2} \dot{\varphi}^{2} g_{a b}+\frac{1}{2} V(\varphi) g_{a b}- \\
& -\frac{1}{2} \varepsilon(\varphi) g_{a b}\left\{12 \ddot{u}_{1}\left(2 \dot{u}_{1}^{2}+(q-1)_{2} \dot{u}_{2}^{2}+4(q-1) \dot{u}_{1} \dot{u}_{2}\right)+4 \ddot{u}_{2}\left(6(q-1)_{2} \dot{u}_{1} \dot{u}_{2}+\right.\right. \\
& \left.+(q-1)_{3} \dot{u}_{2}^{2}+6(q-1) \dot{u}_{1}^{2}\right)+24 \dot{u}_{1}^{4}+q_{3} \dot{u}_{2}^{4}+24(q-1)(2 q-3) \dot{u}_{1}^{2} \dot{u}_{2}^{2}+ \\
& \left.+72(q-1) \dot{u}_{1}^{3} \dot{u}_{2}+12(q-1)(q-1)_{2} \dot{u}_{1} \dot{u}_{2}^{3}\right\}+2 g_{a b}\left\{-\left(\varepsilon^{\prime \prime} \dot{\varphi}^{2}+\varepsilon^{\prime} \ddot{\varphi}\right)\left[6 \dot{u}_{1}^{2}+\right.\right. \\
& \left.+(q-1)_{2} \dot{u}_{2}^{2}+6(q-1) \dot{u}_{1} \dot{u}_{2}\right]+\varepsilon^{\prime} \dot{u}_{2} \dot{\varphi}\left[-6(q-1) \ddot{u}_{1}-2(q-1)_{2} \ddot{u}_{2}-\right. \\
& \left.-12(q-1) \dot{u}_{1}^{2}-(q-1)(q-1)_{2} \dot{u}_{2}^{2}-6(q-1)_{2} \dot{u}_{1} \dot{u}_{2}\right]+\varepsilon^{\prime} \dot{u}_{1} \dot{\varphi}\left[-12 \ddot{u}_{1}-\right. \\
& \left.\left.-6(q-1) \ddot{u}_{2}-18 \dot{u}_{1}^{2}-3 q_{1} \dot{u}_{2}^{2}-12(q-1) \dot{u}_{1} \dot{u}_{2}\right]\right\}=\frac{8 \pi G}{c^{4}} T_{a b} . \\
& +2 \\
& \left.\left.+6(q-1) \dot{u}_{1} \dot{u}_{2}\right]+24 \dot{u}_{1}^{4}+(q+1) q_{2} \dot{u}_{2}^{4}+24 q(2 q-1) \dot{u}_{1}^{2} \dot{u}_{2}^{2}+72 q \dot{u}_{1}^{3} \dot{u}_{2}+12 q q_{1} \dot{u}_{1} \dot{u}_{2}^{3}\right\}=0 . \\
& -2\left(3 \dot{u}_{1}+q \dot{u}_{2}\right) \dot{\varphi}+V^{\prime}(\varphi)-\varepsilon^{\prime}(\varphi)\left\{12 \ddot{u}_{1}\left[2 \dot{u}_{1}^{2}+q_{1} \dot{u}_{2}^{2}+4 q \dot{u}_{1} \dot{u}_{2}\right]+4 q \ddot{u}_{2}\left[6 \dot{u}_{1}^{2}+(q-1)_{2}^{2}+\right.\right.
\end{aligned}
$$

Find at first solutions without dilaton, without matter and with constant Hubble parameters:

$\varphi=0, \quad V(\varphi)=0, \quad T_{\mu v}=0, \quad \dot{u}_{1}=$ const,$\quad \dot{u}_{2}=$ const.

Then system (44)-(46) will be a system of algebraic equations for which we have found two analytical solutions for arbitrary $q$ and negative $\varepsilon$ :

$$
\dot{u}_{1}=\dot{u}_{2}= \pm \frac{1}{\sqrt{-q(q+1) \varepsilon}} .
$$

Also particular cases from $q=1$ to $q=22 \quad(22+4=26$ is required for the bosonic strings, and in the case of $q=0$ Lovelock gravity is just Einstein gravity) have been studied, but solutions different from (49) have been obtained only for $q=3$ (i. e. for 7-dimensional space just as in section 1):

$\dot{u}_{1}=-\zeta_{1} \sqrt{\frac{3+\zeta_{2} \sqrt{5}}{2 \varepsilon}} \cdot \frac{575+257 \zeta_{2} \sqrt{5}}{3010+1346 \zeta_{2} \sqrt{5}}, \quad \dot{u}_{2}=\zeta_{1} \sqrt{\frac{3+\zeta_{2} \sqrt{5}}{8 \varepsilon}}$,

and

$\dot{u}_{1}=\zeta_{1} \sqrt{\frac{3+\zeta_{2} \sqrt{5}}{8 \varepsilon}}, \quad \dot{u}_{2}=-\zeta_{1} \sqrt{\frac{3+\zeta_{2} \sqrt{5}}{2 \varepsilon}} \cdot \frac{575+257 \zeta_{2} \sqrt{5}}{3010+1346 \zeta_{2} \sqrt{5}}$,

where constants $\zeta_{1}$ and $\zeta_{2}$ take values of +1 and -1 independently from each other, and $\varepsilon>0$.

Therefore scale factors are

$$
a(t) \equiv e^{u_{1}}=a_{0} e^{\dot{u}_{1} t}, \quad b(t) \equiv e^{u_{2}}=b_{0} e^{\dot{u}_{2} t} .
$$

It is clear that solutions (49) are not useful for us by the following cause: when visible subspace expands, extra subspace expands too, then extra subspace must be visible in this case. But solutions (50) for $\zeta_{1}=-1$ and (51) for $\zeta_{1}=+1$ satisfy our purpose.

Now let us try to obtain exponential solutions in the presence of perfect fluid. For that substitute conditions (48) by

$$
\begin{aligned}
& \varphi=0, \quad V(\varphi)=0, \quad T_{00}=\varepsilon, \quad T_{i j}=w \varepsilon g_{i j}, \\
& T_{a b}=w \varepsilon g_{a b}, \quad \dot{u}_{1}=\text { const }, \quad \dot{u}_{2}=\text { const } .
\end{aligned}
$$

After plugging those into (44)--(46) and subtracting factors $g_{i j}$ and $g_{a b}$ we see that left-hand sides of equations are independent of time. Hence the right-hand sides also must be constant.

From 00-component of local conservation law for energy-momentum tensor $\left(\nabla^{\mu} T_{\mu 0}=0\right)$ one can obtain (taking (52) into account)

$$
\varepsilon=\varepsilon_{0} \exp \left[-(1+w)\left(3 \dot{u}_{1}+q \dot{u}_{2}\right) t\right] .
$$

Therefore $\varepsilon=\mathrm{const}$ under at least one of a two conditions (here $H \equiv \dot{u}_{1}, h \equiv \dot{u}_{2}$ ):

1. $w=-1$;

2. $h=-\frac{3}{q} H$.

In the first case matter can be described by cosmological constant, in the second one comoving bulk is constant. In the 
latter case equations (44)-(46), as equations on $H, \varepsilon, w$, have two solutions:

1.

$$
H=0, \quad \varepsilon=0, \quad w \text { is arbitrary, }
$$

i. e. flat space with Lorenz metric.

2.

$$
\begin{aligned}
H \text { is arbitrary, } \quad \varepsilon & =\frac{3 c^{4} H^{2}\left(-q^{3}-3 q^{2}+3 \varepsilon H^{2} q^{3}+54 \varepsilon H^{2} q^{2}+81 \varepsilon H^{2} q-162 \varepsilon H^{2}\right)}{16 \pi G q^{3}}, \\
w & =\frac{\varepsilon H^{2} q^{2}-q^{2}+15 \varepsilon H^{2} q-18 \varepsilon H^{2}}{-q^{2}+3 \varepsilon H^{2} q^{2}+45 \varepsilon H^{2} q-54 \varepsilon H^{2}},
\end{aligned}
$$

i. e. one can obtain any value for $H$ by matching energy density $\varepsilon$ and EoS parameter $w$. It is clear that $h<0$ if $H>0$, that's why this solution satisfies all requirements. Finally, such a solution describes anisotropic expansion of the Universe with matter which not tends to isotropization. In Einstein gravity it is possible only for maximally stiff fluid: $w=1$.

Now turn to the cosmological constant case: $w=-1$. Then it is possible to consider (44)-(46) as equations on $H$, $h$ and $\varepsilon$. These have the following solutions:

1.

$$
\begin{aligned}
& H=h \text { and is arbitrary, } \\
& \varepsilon=\frac{c^{4} h^{2}\left(6+q^{2}+5 q+\varepsilon q^{4} h^{2}+6 \varepsilon q^{3} h^{2}+11 h^{2} \varepsilon q^{2}+6 h^{2} \varepsilon q\right)}{16 \pi G} ;
\end{aligned}
$$

2.

$$
\begin{aligned}
& h \text { is arbitrary, } \quad H=-(q-1) h \pm \frac{\sqrt{2 \varepsilon^{2} h^{2} q(q-1)-\varepsilon}}{2 \varepsilon} \\
& \begin{aligned}
\epsilon= & \frac{c^{4}}{32 \varepsilon \pi G}\left(-96 H \varepsilon^{2} q^{2} h^{3}+48 H \varepsilon^{2} q h^{3}-24 H \varepsilon q h+48 H \varepsilon^{2} q^{3} h^{3}+24 H h \varepsilon+2 h^{2} \varepsilon q^{2}+\right. \\
& \left.+10 h^{2} \varepsilon q+14 \varepsilon^{2} q^{4} h^{4}-60 \varepsilon^{2} q^{3} h^{4}+82 \varepsilon^{2} q^{2} h^{4}-36 \varepsilon^{2} q h^{4}-3-12 h^{2} \varepsilon\right) .
\end{aligned}
\end{aligned}
$$

It is evident that the first solution is unsatisfactory. The second one is adequate under $h<0, H>0$. Such conditions are fulfilled in three cases:
Here we also should emphasize an existence of solutions with matter which do not tend to isotropization.

\subsection{Exponent-of-Exponent form Solutions}

Try to obtain solutions with a dynamical dilaton. For that purpose consider equations (44)--(47) and notice that functions $u_{1}(t)$ and $u_{2}(t)$ make contribution only through derivatives $\dot{u}_{1}, \dot{u}_{2}, \ddot{u}_{1}, \ddot{u}_{2}$, but $\varphi(t)$ participate explicitly. To find solutions with constant derivatives let's eliminate $\varphi(t)$ by introducing new time variable. At first we put

$$
\varepsilon(\varphi)=\beta e^{-\gamma \varphi}, \quad V(\varphi)=\alpha e^{\gamma \varphi}, \quad T_{\mu \nu}=0 .
$$

Now turn from time $t$ to new variable $\tau$ :

$$
\partial_{\tau}=e^{-\gamma \varphi / 2} \partial_{t} .
$$

$$
\begin{aligned}
& 3 u_{1}^{\prime 2}+\frac{1}{2} q_{1} u_{2}^{\prime 2}+3 q u_{1}^{\prime} u_{2}^{\prime}+\frac{1}{2} \varphi^{\prime 2}-\frac{1}{2} \alpha+\frac{1}{2} \beta\left\{36 q_{1} u_{1}^{\prime 2} u_{2}^{\prime 2}+q_{3} u_{2}^{\prime 4}+\right. \\
& \left.\quad+12 u_{1}^{\prime} u_{2}^{\prime}\left(2 q u_{1}^{\prime 2}+q_{2} u_{2}^{\prime 2}\right)\right\}-2 \beta \gamma \varphi^{\prime}\left\{18 q u_{1}^{\prime 2} u_{2}^{\prime}+9 q_{1} u_{1}^{\prime} u_{2}^{\prime 2}+6 q u_{1}^{\prime 3}+q_{2} u_{2}^{\prime 3}\right\}=0 \\
& -3 u_{1}^{\prime 2}-\frac{1}{2}(q+1) q u_{2}^{\prime 2}-\gamma \varphi^{\prime}\left(u_{1}^{\prime}+\frac{q}{2} u_{2}^{\prime}\right)-2 q u_{1}^{\prime} u_{2}^{\prime}+\frac{1}{2}{\varphi^{\prime}}^{2}+\frac{\alpha}{2}-\frac{1}{2} \beta\left\{-4 q(3 q+1) \gamma \varphi^{\prime} u_{1}^{\prime} u_{2}^{\prime 2}-\right. \\
& \quad-28 q \gamma \varphi^{\prime} u_{1}^{\prime 2} u_{2}^{\prime}-2(q+2) q_{1} \gamma \varphi^{\prime} u_{2}^{\prime 3}-4 \gamma^{2}{\varphi^{\prime}}^{2} u_{1}^{\prime 2}-2 q_{1} \gamma^{2}{\varphi^{\prime}}^{2} u_{2}^{\prime 2}+4 q(5 q-3) u_{1}^{\prime 2} u_{2}^{\prime 2}+ \\
& \left.+8 q q_{1} u_{1}^{\prime} u_{2}^{\prime 3}+16 q u_{1}^{\prime 3} u_{2}^{\prime}+(q+1) q_{2} u_{2}^{\prime 4}-16 \gamma \varphi^{\prime} u_{1}^{\prime 3}-8 q \gamma^{2} \varphi^{\prime 2} u_{1}^{\prime} u_{2}^{\prime}\right\}=0
\end{aligned}
$$




$$
\begin{aligned}
& -\frac{1}{2} q_{1} u_{2}^{\prime 2}-6 u_{1}^{\prime 2}-\frac{q-1}{2} \gamma \varphi^{\prime} u_{2}^{\prime}-\frac{3}{2} \gamma \varphi^{\prime} u_{1}^{\prime}-3(q-1) u_{1}^{\prime} u_{2}^{\prime}+\frac{1}{2} \varphi^{\prime 2}+\frac{\alpha}{2}- \\
& -\frac{1}{2} \beta\left\{-2(q+1)(q-1)_{2} \gamma \varphi^{\prime} u_{2}^{\prime 3}-6(q-1)(3 q-2) \gamma \varphi^{\prime} u_{1}^{\prime} u_{2}^{\prime 2}-60(q-1) \gamma \varphi^{\prime} u_{1}^{\prime 2} u_{2}^{\prime}-\right. \\
& \quad-60 \gamma \varphi^{\prime} u_{1}^{\prime 3}-2(q-1)_{2} \gamma^{2} \varphi^{\prime 2} u_{2}^{\prime 2}-12 \gamma^{2} \varphi^{\prime 2} u_{1}^{\prime 2}+q_{3} u_{2}^{\prime 4}+24(q-1)(2 q-3) u_{1}^{\prime 2} u_{2}^{\prime 2}+ \\
& \left.\quad+12(q-1)(q-1)_{2} u_{1}^{\prime} u_{2}^{\prime 3}+72(q-1) u_{1}^{\prime 3} u_{2}^{\prime}+24 u_{1}^{\prime 4}-12(q-1) \gamma^{2} \varphi^{\prime 2} u_{1}^{\prime} u_{2}^{\prime}\right\}=0 \\
& -\gamma \varphi^{\prime 2}-2\left(3 u_{1}^{\prime}+q u_{2}^{\prime}\right) \varphi^{\prime}+\beta \gamma\left\{12 q(4 q-2) u_{1}^{\prime 2} u_{2}^{\prime 2}+(q+1) q_{2} u_{2}^{\prime 4}+72 q u_{1}^{\prime 3} u_{2}^{\prime}+12 q q_{1} u_{1}^{\prime} u_{2}^{\prime 3}+\right. \\
& \left.\quad+18 q_{1} \gamma \varphi^{\prime} u_{1}^{\prime} u_{2}^{\prime 2}+36 q \gamma \varphi^{\prime} u_{1}^{\prime 2} u_{2}^{\prime}+12 \gamma \varphi^{\prime} u_{1}^{3}+24 u_{1}^{4}+2 q_{2} \gamma \varphi^{\prime} u_{2}^{\prime 3}\right\}+\alpha \gamma=0 .
\end{aligned}
$$

Now assume that we have obtained some quantities $u_{1}^{\prime}, u_{2}^{\prime}, \varphi^{\prime}$ which satisfy these equations. What should they be to describe accelerating expansion of visible subspace and contraction of extra one? It is easy to see that the scale factor of visible subspace would be

$$
a(t) \equiv e^{u_{1}^{\prime}}=a_{0} \exp \left\{\frac{2 u_{1_{1}} c_{0}}{\varphi^{\prime}} e^{\varphi^{\prime} t / 2}\right\},
$$

where $a_{0}, c_{0}$ are arbitrary positive constants. Then its first and second derivatives with respect to time $t$ would be
Finally, field equations in the case of flat subspaces without matter and with $V(\varphi)$ and $\varepsilon(\varphi)$ in the form of (56) have exponent-of-exponent form solutions (61), (62) with abovementioned parameters.

\subsection{Power-Law Solutions}

Now consider space with dust-like matter:

$$
T_{00} \neq 0, \quad \text { other } T_{\mu v}=0 .
$$

And try to obtain solutions of system (44)--(47) with scale factors of power-law form:

$$
\begin{aligned}
& \dot{a}(t)=a_{0} u_{1}^{\prime} c_{0} e^{\varphi^{\prime} t / 2} \exp \left\{\frac{2 u_{1}^{\prime} c_{0}}{\varphi^{\prime}} e^{\varphi^{\prime} t / 2}\right\}, \\
& \ddot{a}(t)=\frac{1}{2} a_{0} u_{1}^{\prime} c_{0} \varphi^{\prime} e^{\varphi^{\prime} t / 2} \exp \left\{\frac{2 u_{1}^{\prime} c_{0}}{\varphi^{\prime}} e^{\varphi^{\varphi^{\prime}} t / 2}\right\}+a_{0} u_{1}^{\prime}{ }_{1}^{2} c_{0}^{2} e^{\varphi^{\prime} t} \exp \left\{\frac{2 u_{1}^{\prime} c_{0}}{\varphi^{\prime}} e^{\varphi^{\prime} t / 2}\right\} .
\end{aligned}
$$

It is clear that both derivatives would be positive (as need for accelerating expansion) if $u_{1}^{\prime}>0, \varphi^{\prime}>0$ (the latter is not necessary but is sufficient).

By the same manner we obtain the scale factor for extra dimensions:

$$
b(t) \equiv e^{u^{\prime} 2}=b_{0} \exp \left\{\frac{2 u_{2}^{\prime} c_{0}}{\varphi^{\prime}} e^{\varphi^{\prime} t / 2}\right\}
$$

( $b_{0}$ is positive constant) and its first derivative:

$$
\dot{b}(t)=b_{0} u_{2}^{\prime} c_{0} e^{\varphi^{\prime} t / 2} \exp \left\{\frac{2 u_{2}^{\prime} c_{0}}{\varphi^{\prime}} e^{\varphi^{\prime} t / 2}\right\},
$$

which would be negative (as need for contraction) under $u_{2}^{\prime}<0$.

Therefore it is necessary to find solutions of (57)-(60) satisfied conditions

$u_{1}^{\prime}>0, \quad u_{2}^{\prime}<0, \quad \varphi^{\prime}>0$.

Numerical calculations give us solutions for different dimensions from $q=1$ to $q=20$. For example,

$$
\begin{array}{ll}
q=1, & \alpha=1, \beta=1, \gamma=1, \\
q=2, & \alpha=0.01, \beta=100, \gamma=0.01, \\
q=3, & \alpha=0.001, \beta=100, \gamma=0.001,
\end{array}
$$

$$
a(t) \equiv e^{u_{1}}=\left(\frac{t}{t_{1}}\right)^{n}, \quad b(t) \equiv e^{u_{2}}=\left(\frac{t}{t_{2}}\right)^{m}
$$

Then all Einstein terms in left-hand sides of equations (44)-(46) are proportional to $1 / t^{2}$ and Gauss-Bonnet terms are proportional to $1 / t^{4}$. Hence solutions with scale factors (63) are possible if

$$
\begin{gathered}
T_{00} \propto \frac{1}{t^{2}}, \\
\dot{\varphi}^{2} \propto \frac{1}{t^{2}}, \\
\varepsilon(\varphi) \propto t^{2}, \\
V(\varphi) \propto \frac{1}{t^{2}},
\end{gathered}
$$

Consider these conditions one by one.

It is possible to derive time dependence of energy density from conservation law of energy-momentum tensor:

$$
T^{00}=\operatorname{const} \cdot t^{-3 n-q m} .
$$

$\varphi^{\prime}=0.383$

$u_{1}^{\prime}=0.378$

$u_{2}^{\prime}=-1.32$.

$\varphi^{\prime}=5.09 \cdot 10^{-5}$,

$u_{1}^{\prime}=0.0175$,

$u_{2}^{\prime}=-0.0803$.

$\varphi^{\prime}=2.33 \cdot 10^{-4}$,

$u_{1}^{\prime}=0.0306$,

$u_{2}^{\prime}=-0.0788$. 
From comparison this expression with (64) we have

$$
m=\frac{2-3 n}{q} .
$$

Note that under this condition extra subspace contracts $(m<0)$ if visible subspace expanses accelerative $(n>1)$ (but we haven't obtain such a solution, see below). For (3+1)-dimensional space condition (64) leads to $n=2 / 3 \mathrm{i}$. e. to Friedmann solution.

From condition (65) it is easy to obtain $\varphi(t)=\psi \ln \left(t / t_{3}\right)$, where $\psi, t_{3}$ are arbitrary constants $\left(t_{3}>0\right)$. In order to avoid unnecessary complication put $\psi=1$. Therefore

$$
\varphi(t)=\ln \frac{t}{t_{3}}
$$

From comparison this expression with (66), (67) we see:

$$
V(\varphi)=\tilde{\alpha} e^{-2 \varphi}, \quad \varepsilon(\varphi)=\tilde{\beta} e^{2 \varphi},
$$

where $\tilde{\alpha}, \tilde{\beta}$ are constants.

Plugging all those into equations (44)-(47) and putting

$$
\tilde{\alpha} \rightarrow \alpha=\tilde{\alpha} t_{3}^{2}, \quad \tilde{\beta} \rightarrow \beta=\tilde{\beta} / t_{3}^{2},
$$

one can obtain a system of algebraic equations on $n$ with parameters $\alpha, \beta, q$ and $\varkappa \equiv \frac{8 \pi G}{c^{4}} T_{00}\left(t_{0}\right) t_{0}^{2}$, where $T_{00}\left(t_{0}\right)$ is energy density at some time moment $t_{0}$. Such a system of equations has solutions not at all values of parameters. Considering dimensions from $q=1$ to $q=22$ we have obtained a set of solutions with arbitrary $\mathcal{X}$. Here $\alpha$ and $\beta$ are functions of $\mathcal{X}$ and $0<n=m<1$ i. e. visible and extra subspaces expands with deceleration (and with the same velocity). In another set of solutions $\mathcal{X}$ possesses fixed values. Here in two cases $n \neq$ $m$ and in another cases $n$ These are solutions for $q=1,2,6$, 9, 12:

Note that in the last two cases $\beta=0$, therefore these solutions are solutions in Brans-Dicke theory (i. e. theory with Lagrangian (20) without Gauss-Bonnet term).
In all obtained power-law solutions $0<n<1,0<m<1$, that's why such solutions don't describe accelerated expansion of visible space or contraction of extra dimensions. However solutions with $n \neq m$ are interesting for another cause. In Einstein theory there is no anisotropic power-law solution in the presence of dust. However, in Einstein-GaussBonnet theory with dilaton that is possible.

\section{CONCLUSION}

Different variants of Lovelock gravity with dilaton were considered in $D$-dimensional space with two maximally symmetric subspaces: 3-dimensional and (D-4)dimensional. Absence of matter and existence of perfect fluid were investigated. We have several types of obtained solutions:

Stationary.

Power-law.

Exponential.

Exponent-of-exponent form solutions.

Among the last two forms solutions which describe accelerating expansion of 3-dimensional subspace and contraction of $(D-4)$-dimensional one were elected. Unobservability of the latter subspace was justified on the basis of KaluzaKlein approach. Also a set of anisotropic solutions which do not tend to isotropization in the presence of matter, in contrast to Einstein gravity, have been obtained. Such a possibility is of importance because it allows us to assume that extra dimensions become small during the Universe evolution. This issue we are going to investigate in more detail in another work. Moreover, it would be interesting to extend the results of this work for account of third-order Lovelock terms. This will be done elsewhere.

Studying of future singularities in such models would also be important. For 4-dimensional modified gravities this problem was considered in [27, 28].

Unfortunately most of solutions describe only flat maximally symmetric subspaces. For curved subspaces there are

$$
\begin{gathered}
q=1, \quad \varkappa \text { is arbitrary, } \quad \alpha=\frac{3}{7} \varkappa-\frac{29}{14}, \quad \beta=\frac{2}{21} \varkappa-\frac{5}{21}, \quad n=m=\frac{1}{2} . \\
q=2, \quad \varkappa=\frac{53}{40}, \quad \alpha=-\frac{973}{640}, \quad \beta=-\frac{25}{272}, \quad n=\frac{3}{10}, \quad m=\frac{11}{20} . \\
q=6, \quad \varkappa \text { is arbitrary, } \quad \alpha=\frac{112}{253} \varkappa-\frac{5077}{2277}, \quad \beta=\frac{729}{16192} \varkappa-\frac{2025}{16192}, \quad n=m=\frac{2}{9} . \\
q=6, \quad \alpha=\frac{51397}{39528}, \quad \alpha=-\frac{21035}{13176}, \quad \beta=-\frac{243}{3904}, \quad n=\frac{8}{27}, \quad m=\frac{5}{27} . \\
q=9, \quad \varkappa=\frac{17}{6}, \quad \alpha=-1, \quad \beta=0, \quad n=m=\frac{1}{6} . \\
\quad \varkappa=12, \quad \alpha=\frac{43}{15}, \quad \alpha=-1, \quad \beta=0, \quad n=m=\frac{2}{15} .
\end{gathered}
$$


only stationary solutions. Those are of interest as exact solutions of very complicated equations and as possible basis for numerical dynamical solutions in the case of curved subspaces.

\section{ACKNOWLEDGEMENTS}

This work is partially supported by RFBR grant 06-0100609 and by RF Presidential grant for LSS 2553.2008.2. I.V.K. is supported by grants of Tomsk State University academic council and of "Dynasty" foundation in the frameworks of International Center for Fundamental Physics in Moscow. The authors are grateful to S. D. Odintsov, K. E. Osetrin and A. V. Toporensky for useful discussions.

\section{REFERENCES}

[1] Kowalski M, Rubin D, Aldering G, et al. Improved cosmological constraints from new, old and combined supernova datasets. 4 July 2008: Available from: http://xxx.lanl.gov/abs/0804.4142

[2] Bernstein GM. Comprehensive two-point analyses of weak gravitational lensing surveys. 12 February 2009: Available from: http://xxx.lanl.gov/abs/0808.3400

[3] Brans CH, Dicke RH. Mach's principle and a relativistic theory of gravitation. Phys Rev 1961; 124: 925-35.

[4] Dicke RH. Mach's principle and invariance under transformation of units. Phys Rev 1962; 125: 2163-67.

[5] Lovelock D. The einstein tensor and its generalizations. J Math Phys 1971; 12: 498-501.

[6] Nojiri S, Odintsov SD. Introduction to modified gravity and gravitational alternative for dark energy. Int J Geometric Methods Phys 2007; 4: 115-46.

[7] Nojiri S, Odintsov SD, Sasaki M. Gauss-Bonnet dark energy. 17 May 2008; Available from: http://xxx.lanl.gov/abs/hep-th/0504052

[8] Nojiri S, Odintsov SD. Modified Gauss-Bonnet theory as gravitational alternative for dark energy. 15 May 2008; Available from: http://xxx.lanl.gov/abs/hep-th/0508049

[9] Elizalde E, Makarenko AN, Obukhov VV, Osetrin KE, Filippov AE. Stationary $v s$. singular points in an accelerating FRW cosmology derived from six-dimensional Einstein Gauss-Bonnet gravity. 23 August 2008; Available from: http://xxx.lanl.gov/abs/hepth/ 0611213

[10] Dehghani MH, Shamirzaie M. Thermodynamics of asimptotically flat charged black holes in third order lovelock gravity. 3 March 2008; Available from: http://xxx.lanl.gov/abs/hep-th/0506227

[11] Dehghani MH, Mann RB. Thermodynamics of rotating charged black branes in third order lovelock gravity and the counterterm method. 3 March 2008; Available from: http://xxx.lanl.gov/abs/ hep-th/0602243
[12] Dehghani MN, Bostani N. Spacetimes with longitudinal and angular magnetic fields in third order lovelock gravity. 3 March 2008; Available from: http://xxx.lanl.gov/abs/hep-th/0612103

[13] Kirnos IV, Makarenko AN, Osetrin KE. Cosmological solutions in the Lovelock theory and the Einstein-Gauss-Bonnet theory with a dilaton. Grav Cosmol 2009; 19: 59-61.

[14] Cognola G, Elizalde E, Nojiri S, Odintsov SD, Zerbini S. Stringinspired Gauss-Bonnet gravity reconstructed from the universe expansion history and yielding the transition from matter dominance to dark energy. 7 August 2008; Available from: http://xxx.lanl.gov/abs/hep-th/0611198

[15] Bamba K, Guo ZK, Ohta N. Accelerating cosmologies in the einstein-gauss-bonnet theory with dilaton. Prog Theor Phys 2007; 118 : 879-92.

[16] Briggs CC. A general expression for the quartic lovelock tensor. 21 April 2008; Available from: http://xxx.lanl.gov/abs/gr-qc/9703074

[17] Briggs CC. A general expression for the quintic lovelock tensor. 21 April 2008; Available from: http://xxx.lanl.gov/abs/gr-qc/9607033

[18] Cognola G, Elizalde E, Nojiri S, Odintsov SD, Zerbini S. Dark energy in modified Gauss-Bonnet gravity: late-time acceleration and the hierarchy problem. 25 October 2008; Available from: http://xxx.lanl.gov/abs/hep-th/0601008

[19] Nojiri S, Odintsov SD, Sami M. Dark energy cosmology from higher-order string-inspired gravity and its reconstruction. $25 \mathrm{Oc}$ tober 2008; Available from: http://xxx.lanl.gov/abs/hep-th/0605039

[20] Elizalde E, Jhingan S, Nojiri S, Odintsov SD, Sami M, Thongkool I. Dark energy generated from a (super)string effective action with higher order curvature corrections and a dynamical dilaton. 23 August 2008; Available from: http://xxx.lanl.gov/abs/hepth/ 0705.1211

[21] Carroll SM. Spacetime and geometry: an introduction to general relativity. Addison Wesley: San Francisco 2004

[22] Kasner E. Geometrical theorems on Einstein's cosmological equations. Am J Math 1921; 43: 217.

[23] Landau LD, Lifshitz EM. The classical theory of fields. Oxford Pergamon Press: 2002.

[24] Einstein A. Kosmologische Betrachtungen zur allgemeinen Relativitdtstheorie. Sitzungsber preuss Akad Wiss 1917; 1: 142-52.

[25] Jaffe AH, Ade PAR, Balbi A, et al. Cosmology from MAXIMA-1, BOOMERANG and COBE/DMR cosmic microwave background observations. Phys Rev Lett 2001; 86: 3475-79.

[26] Spergel DN, Verde L, Peiris HV, et al. First year wilkinson microwave anisotropy probe (WMAP) observations: determination of cosmological parameters. Astrophys J Suppl 2003; 148: 175.

[27] Nojiri S, Odintsov SD. The future evolution and finite-time singularities in $f(R)$ gravity unifying the inflation and cosmic acceleration. 14 November 2008; Available from: http://xxx.lanl.gov/abs/ hep-th/0804.3519

[28] Bamba K, Nojiri S, Odintsov SD. The universe future in modified gravity theories: approaching the finite-time future singularity. 14 November 2008; Available from: http://xxx.lanl.gov/abs/hepth/0807.2575

Received: August 20, 2009

Revised: September 23, 2009

Accepted: September 23, 2009

(c) Kirnos and Makarenko; Licensee Bentham Open.

This is an open access article licensed under the terms of the Creative Commons Attribution Non-Commercial License (http://creativecommons.org/licenses/by-nc/3.0/) which permits unrestricted, non-commercial use, distribution and reproduction in any medium, provided the work is properly cited. 